\title{
Long-term immunogenicity and safety after a single dose of the quadrivalent meningococcal serogroups $A, C, W$, and $Y$ tetanus toxoid conjugate vaccine in adolescents and adults: 5-year follow-up of an open, randomized trial
}

Charissa Fay Corazon Borja-Tabora ${ }^{\text {* }}$, Cecilia Montalban², Ziad A. Memish ${ }^{3}$, Dominique Boutriau ${ }^{4}$, Devayani Kolhe ${ }^{5}$, Jacqueline M. Miller ${ }^{6}$ and Marie Van der Wielen ${ }^{4}$

\begin{abstract}
Background: Long-term protection against meningococcal disease is associated with persistence of post-vaccination antibodies at protective levels. We evaluated the bactericidal antibody persistence and safety of the quadrivalent meningococcal serogroups A, C, W and $Y$ tetanus-toxoid conjugate vaccine (MenACWY-TT) and the meningococcal polysaccharide serogroups A, C, W, and $Y$ vaccine (MenACWY-PS) up to 5 years post-vaccination.
\end{abstract}

Methods: This phase IIb, open, randomized, controlled study conducted in the Philippines and Saudi Arabia consisted of a vaccination phase and a long-term persistence phase. Healthy adolescents and adults aged 11-55 years were randomized (3:1) to receive a single dose of MenACWY-TT (ACWY-TT group) or MenACWY-PS (Men-PS group). Primary and persistence results up to 3 years post-vaccination have been previously reported. Antibody responses against meningococcal serogroups $A, C, W$, and $Y$ were assessed by a serum bactericidal antibody assay using rabbit complement (rSBA, cut-off titers 1:8 and 1:128) at Year 4 and Year 5 post-vaccination. Vaccine-related serious adverse events (SAEs) and cases of meningococcal disease were assessed up to Year 5.

Results: Of the 500 vaccinated participants, 404 returned for the Year 5 study visit (Total Cohort Year 5). For the Total Cohort Year 5, 71.6-90.0 and 64.9-86.3\% of MenACWY-TT recipients had rSBA titers $\geq 1: 8$ and $\geq 1: 128$, respectively, compared to 24.8-74.3 and 21.0-68.6 \% of MenACWY-PS recipients. The rSBA geometric mean titers (GMTs) remained above the pre-vaccination levels in both treatment groups. Exploratory analyses suggested that both rSBA GMTs as well as the percentages of participants with rSBA titers above the cut-offs were higher in the ACWY-TT than in the Men-PS group for serogroups $A, W$ and $Y$, with no apparent difference for MenC. No SAEs related to vaccination or cases of meningococcal disease were reported up to Year 5.

Conclusion: These results suggest that a single dose of MenACWY-TT could protect at least $72 \%$ of vaccinated adolescents and adults against meningococcal disease at least 5 years post-vaccination.

Trial registration: ClinicalTrials.gov NCT00356369

Keywords: Quadrivalent meningococcal conjugate vaccine, Bactericidal antibody, Antibody persistence, Safety

\footnotetext{
* Correspondence: cbtabora@yahoo.com

'Research Institute for Tropical Medicine, Corporate Ave, Muntinlupa City

1781Metro Manila, Philippines

Full list of author information is available at the end of the article
}

C Biomed Central (c) 2015 Borja-Tabora et al. Open Access This article is distributed under the terms of the Creative Commons Attribution 4.0 International License (http://creativecommons.org/licenses/by/4.0/), which permits unrestricted use, distribution, and reproduction in any medium, provided you give appropriate credit to the original author(s) and the source, provide a link to the Creative Commons license, and indicate if changes were made. The Creative Commons Public Domain Dedication waiver (http://creativecommons.org/publicdomain/zero/1.0/) applies to the data made available in this article, unless otherwise stated. 


\section{Background}

Neisseria meningitidis serogroups A, B, C, W, Y and X account for the majority of invasive meningococcal infections, which are associated with high morbidity and mortality rates [1-3]. There are both geographical and temporal variations in the distribution of these serogroups; these variations are potentially influenced by international travel patterns [1-4]. The risk for infection is higher during disease outbreaks and for people living in or traveling to areas with a higher incidence of endemic disease (e.g. the meningitis belt of Africa) [5-7]. In Asia and the Middle East, serogroups A, C and W are predominant [2]. In the Philippines, serogroup A was responsible for an outbreak of meningococcal disease in 2004-2005 [8]. In Saudi Arabia, outbreaks of meningococcal disease associated with mass gatherings during the Hajj or Umrah pilgrimages facilitating person-to-person transmission have repeatedly occurred [9-11]. Serogroup A was responsible for an outbreak following the Hajj in 1987 [12], while serogroup W was predominant during outbreaks in 2000 and 2001 [4, 9, 12].

To reduce the burden of disease, meningococcal polysaccharide (MenPS) vaccines were successfully introduced more than 3 decades ago [13]. However, MenPS vaccines have some limitations, including poor immunogenicity in infants and toddlers, a lack of capacity to induce long-term protection or immune memory, a negligible impact on nasopharyngeal carriage, failure to confer herd immunity, and observed hyporesponsiveness after repeated doses $[14,15]$. To overcome these drawbacks, capsular polysaccharides can be coupled to carrier proteins as demonstrated by monovalent meningococcal serogroup C conjugate vaccines [16-20]. Moreover, vaccination against multiple serogroups may be the best strategy to protect individuals against a broader range of meningococcal diseases in a single injection.

Currently, three quadrivalent meningococcal conjugate vaccines (MenACWY) are available [15]: a tetanus toxoid (TT) conjugate vaccine (MenACWY-TT; Nimenrix ${ }^{\mathrm{T}}$, GSK Vaccines, Rixensart, Belgium); a diphtheria toxoid (DT) conjugate vaccine (MenACWY-DT; Menactra ${ }^{\mathrm{TM}}$, Sanofi Pasteur Inc., Swiftwater, Pennsylvania); and a nontoxic mutant variant of Corynebacterium diphtheriae toxin $\left(\mathrm{CRM}_{197}\right)$ conjugate vaccine (MenACWY-CRM; Menveo $^{\mathrm{TM}}$, GSK Vaccines, Rixensart, Belgium). Previous clinical studies have shown that MenACWY-TT is immunogenic and well-tolerated in toddlers, children, adolescents, and adults, and that functional antibodies induced by a single vaccine dose persist up to 3 years after vaccination [21-35].

In both the Philippines and Saudi Arabia, meningococcal vaccination is recommended for individuals who are at increased risk for meningococcal infection. After the 2000 and 2001 disease outbreaks, Saudi Arabia changed the vaccination recommendation from a bivalent meningococcal serogroup $\mathrm{A}$ and $\mathrm{C}$ polysaccharide vaccine to the quadrivalent $(\mathrm{A}, \mathrm{C}, \mathrm{W}$ and $\mathrm{Y}$ ) polysaccharide vaccine as a Hajj visa requirement $[4,36,37]$. No further Hajj or Umrah related outbreaks have occurred since then, and the incidence of invasive meningococcal disease was reduced in the region [12, 36, 38]. Since January 2013, routine immunization of children older than 2 years of age and vaccination of Hajj pilgrims with quadrivalent MenPS or conjugate vaccines is recommended in Saudi Arabia [39]. Hajj pilgrims are required to have the meningococcal vaccine $\leq 3$ years and $\geq 10$ days before arriving in Saudi Arabia. Since individuals may participate in multiple pilgrimages, the conjugate vaccine is preferred to the MenPS vaccine due to concerns of hyporesponsiveness with repeated doses [40]. In the Philippines, meningococcal vaccines are not included in the national routine immunization schedule, but vaccination with quadrivalent polysaccharide (single dose) or conjugate vaccines (single dose in adults, 2 doses in children aged $\geq 9$ months given at 2-month intervals; boosters at 5-year intervals) is recommended by both pediatric and adult infectious disease societies for individuals who are at increased risk for meningococcal infection [41].

The introduction of quadrivalent meningococcal conjugate vaccines has led to the reduction of the number of cases of invasive disease. However, several studies have shown that serum antibody levels elicited by conjugate vaccines wane over time, especially at younger ages [42-44]. Previous clinical data have shown that the ability of meningococcal serogroup $C$ conjugate vaccines to induce immunologic memory does not necessarily confer protection, because the onset of invasive meningococcal disease may occur before sufficient antibodies are produced $[16,45]$. Therefore, the maintenance of persisting antibodies may be a more reliable predictor than immune memory for long-term protection against meningococcal disease [45].

Results of a randomized study enrolling 500 participants showed MenACWY-TT to be non-inferior compared to a licensed MenACWY plain polysaccharide vaccine (MenACWY-PS; Mencevax ${ }^{\mathrm{TM}}$, GSK Vaccines, Belgium) in terms of rSBA vaccine response to the four meningococcal serogroups one month after vaccination. The primary outcome of the study was thus met and results showing that a single dose of MenACWY-TT induced bactericidal antibodies which persisted in a majority of participants, and was well-tolerated up to 3 years post-vaccination were reported in a previous publication [30]. The secondary outcomes described in this publication include the comparison of the long-term persistence of immunogenicity of the two vaccines, and the description of serious adverse events (SAEs) related to vaccination and of any event related to the lack of vaccine efficacy up to Year 5 after vaccination. 


\section{Methods}

\section{Study design and participants}

This phase IIb, open, randomized, controlled study was conducted between December 2006 and February 2013 in two centers in the Philippines (Research Institute for Tropical Medicine in Muntilupa City and Philippine General Hospital in Manila) and one center in Saudi Arabia (King Abdulaziz Medical City in Riyadh). The study consisted of two phases: the vaccination phase (www.clinicaltrials.gov, NCT00356369) and the longterm persistence phase (up to 5 years after vaccination).

Study participants were healthy adolescents and adults aged 11-55 years at the time of vaccination, who had completed routine childhood vaccination to the best of their or their parents'guardians' knowledge. At each yearly visit, participants were excluded from the long-term persistence phase of this study if they had received a meningococcal vaccine not permitted in the protocol or if they had a history of meningococcal disease. Participants with a suboptimal response to all four serogroups at a postvaccination timepoint received an additional vaccine dose and were excluded from the study. Participants with a suboptimal response to one of the four serogroups were not excluded from the study, unless the study investigator considered that the epidemiological risks warranted the administration of an additional vaccine dose.

In the vaccination phase, participants were randomized (3:1) to two groups receiving either a single dose of MenACWY-TT (ACWY-TT group) or MenACWY-PS (Men-PS group); the randomization procedure, accounting for study center and age of the participant (11-17 and 18-55 years of age), as well as the composition and administration of these vaccines, has been previously described [30]. Here, we present results at Years 4 and 5 after vaccination, evaluated between January 2011 and February 2013.

The study was conducted in accordance with the Good Clinical Practice Guidelines and the Declaration of Helsinki, and the protocol and associated documents were reviewed and approved by the following local ethics committees: the Research Institute for Tropical Medicine (RITM) Institutional Review Board, Muntinlupa City, Philippines; University of the Philippines Manila Research Ethics Board, Manila, Philippines; and the National Guard Health Affair Institutional Review Board (IRB), King Abdulaziz Medical City, Riyadh, Kingdom of Saudi Arabia. Written informed consent was obtained before enrolment from the participants aged at least 16 years in Saudi Arabia and 18 years in the Philippines. Younger participants signed a written informed assent and a written informed consent was obtained from their parents/guardians. In addition, consent was obtained if participants reached 18 years of age during the study. A summary of the protocol is available at http:// www.gsk-clinicalstudyregister.com (GSK study ID 107386).

\section{Immunogenicity assessments}

Blood samples were collected from all participants at Years 4 and 5. Functional antibody responses against meningococcal serogroups $\mathrm{A}, \mathrm{C}, \mathrm{W}$ and $\mathrm{Y}$ were assessed by a serum bactericidal activity assay with rabbit complement as exogenous complement source (rSBA, cutoff titer 1:8) [46]. rSBA titers $\geq 1: 8$ correlate with shortterm protection for serogroup C [47], and this threshold was extended to the other serogroups. The percentages of participants with rSBA titers $\geq 1: 128$, a more conservative correlate of protection, were also evaluated [48]. The assay for Years 4 and 5 was performed at the Public Health England (PHE) laboratory. This prevented a direct comparison with the results from earlier timepoints, which were assessed at the GSK Vaccines laboratory, for the entire study cohort. For a subset of participants, samples from previous timepoints were retested at the PHE laboratory for direct comparison.

\section{Safety assessments}

During the long-term persistence phase, only SAEs that were considered by the investigator to have a causal relationship to the vaccination were collected retrospectively up to Year 5. The occurrence of meningococcal disease, as an event related to the lack of vaccine efficacy, was also described. Any pregnancy complication or elective termination of a pregnancy for medical reasons or spontaneous abortion in a study participant was recorded.

\section{Statistical analyses}

Two cohorts were defined for the purpose of the antibody persistence analysis. The Total Cohort Year 5 included all vaccinated participants who attended the Year 5 follow-up visit and for whom data concerning persistence endpoint measures were available. The according-to-protocol (ATP) cohort for persistence Year 5 included all evaluable participants who received the vaccine during the vaccination phase, had not received a previous dose of meningococcal serogroup A, C, W or $\mathrm{Y}$ vaccines not planned in the protocol, were compliant with protocol-defined windows for serum sampling ( 254 to 266 weeks post-vaccination), and had available assay results for at least one tested antigen at the Year 5 timepoint.

The percentages of participants with antibody titers above the proposed cut-offs and the geometric mean antibody titers (GMTs) for the tested antigens were calculated with $95 \%$ confidence intervals (CIs). The exact $95 \%$ CIs for proportion within a group were calculated based on the method described by Clopper et al. [49]. The standardized asymptotic $95 \%$ CIs for the group difference in proportions were calculated based on the method 6 described by Newcombe [50]. Antibody titers below the cut-off of the assay (1:8) were given an arbitrary 
value of half the cut-off for the purpose of GMT calculation.

All comparative analyses between groups were exploratory. Potential differences between groups were identified if the standardized asymptotic $95 \%$ CI for (1) the difference in percentage of participants with titers above the proposed cut-offs between the two groups did not contain the value ' 0 ' or (2) the GMT ratio between the two groups did not contain the value ' 1 '. Since no adjustment for multiplicity of these comparisons was performed, the results should be interpreted with caution.

The analyses were performed using Statistical Analysis System version 9.22 for Windows (SAS Institute Inc., Cary, NC, United States).

\section{Results}

\section{Demographics}

Of the 500 participants who were vaccinated in the primary phase of the study, 426 returned for the Year 4 study visit and 404 for the Year 5 visit. The ATP cohort for persistence Year 4 included 420 participants (313 in the ACWY-TT group and 107 in Men-PS group) and the Year 5 ATP cohort included 70 participants (51 in the ACWY-TT group and 19 in Men-PS group) (Fig. 1). This low number of participants included in the ATP cohort at Year 5 was due to a delay in the annual approval from the Food and Drug Administration (FDA) of the Philippines for the Year 5 persistence timepoint, which caused all 333 participants enrolled in the Philippines to attend the Year 5 visit outside the protocol-defined windows for serum sampling (254 to 266 weeks post-vaccination); they were therefore eliminated from the ATP analysis. In the Total Cohort Year 5, the mean interval between vaccination and the Year 5 serum sampling was $\approx 288$ weeks (range 258 to 301 weeks) post-vaccination.

At the time of vaccination, 301 participants were 1117 years old and 199 participants were 18-55 years old. The age and gender distribution of the participants in the Total Cohort Year 5 were similar in both groups, and were consistent with those of the participants enrolled in the vaccination phase of the study (Table 1). The population in the Total Cohort Year 5 was predominantly of South East Asian heritage (82.4 \%).

\section{Persistence of bactericidal antibodies}

The primary analysis of immunogenicity was based on the ATP cohort for persistence Year 5 (Additional file 1: Table S1). Because none of the participants from the Philippines were included in this cohort due to noncompliance with the blood sampling schedule, the Total Cohort Year 5 was considered to be more representative for the study population, and the results based on this cohort are presented in this manuscript.
In the Total Cohort Year 5, the percentages of participants with seroprotective rSBA titers, as measured by the PHE assay at Year 5, ranged from 71.6 to $90.0 \%$ in the ACWY-TT group, and from 24.8 to $74.3 \%$ in the Men-PS group (Table 2). The percentages of participants with rSBA titers $\geq 1: 128$ ranged from 64.9 to $86.3 \%$ in the ACWY-TT group and from 21.0 to $68.6 \%$ in the Men-PS group. At Year 4, 5 participants (3 from the ACWY-TT group, 2 from the Men-PS group) and at Year 5, 3 participants (1 from ACWY-TT group, 2 from the Men-PS group) had a suboptimal response to all 4 serogroups. These participants received an additional dose of a licensed meningococcal vaccine and were excluded from the long-term follow-up of that particular year and the following years.

The results from the subset of samples retested at the PHE laboratory (collected at pre-vaccination, Year 1 and Year 2) suggested that the largest decrease in rSBA GMTs occurred during the first year following vaccination in both groups for all four serogroups (Table 2). Between Month 1 and Year 5, the rSBA GMTs decreased between 12.0- and 60.4-fold in the ACWY-TT group and between 14.1- to 90.0-fold in the Men-PS group. All rSBA GMTs at Year 5 were still above the pre-vaccination levels determined in the subset of samples retested at the PHE laboratory.

Exploratory analysis at Year 5 suggested that the percentages of participants with rSBA titers $\geq 1: 8$ and $\geq 1: 128$ and the rSBA GMTs for serogroups $\mathrm{A}, \mathrm{W}$ and $\mathrm{Y}$ were significantly higher in the ACWY-TT group than in the Men-PS group (Table 2).

The data for each of the age strata were consistent with the data for the total population: in the ACWY-TT group, the percentage of adolescents (11-17 years of age) with rSBA titers $\geq 1: 8$ was $92.8,80.3,74.0$, and $81.3 \%$ for serogroups $\mathrm{A}, \mathrm{C}, \mathrm{W}$ and $\mathrm{Y}$, respectively, while the percentage of adults (18-55 years of age) with rSBA titers $\geq 1: 8$ was $83.5,76.9,65.9$, and $91.2 \%$ for these serogroups, respectively; in the Men-PS group, the percentage of adolescents with rSBA titers $\geq 1: 8$ was $80.3,65.8,23.7$, and $42.1 \%$ for serogroups $\mathrm{A}, \mathrm{C}, \mathrm{W}$ and $\mathrm{Y}$, respectively, while the percentage of adults with rSBA titers $\geq 1: 8$ was 58.6, 85.7, 27.6, and $51.7 \%$ for serogroups $\mathrm{A}, \mathrm{C}, \mathrm{W}$ and $\mathrm{Y}$, respectively (Additional file 1: Table S2).

\section{Safety}

No SAEs related to vaccination or events related to the lack of efficacy were reported between Month 0 and Year 5 .

Twenty-three study participants reported pregnancies between Year 3 and Year 5; one participant was diagnosed with eclampsia. All pregnancies had a normal outcome and resulted in birth of live infants with no apparent congenital anomalies. 


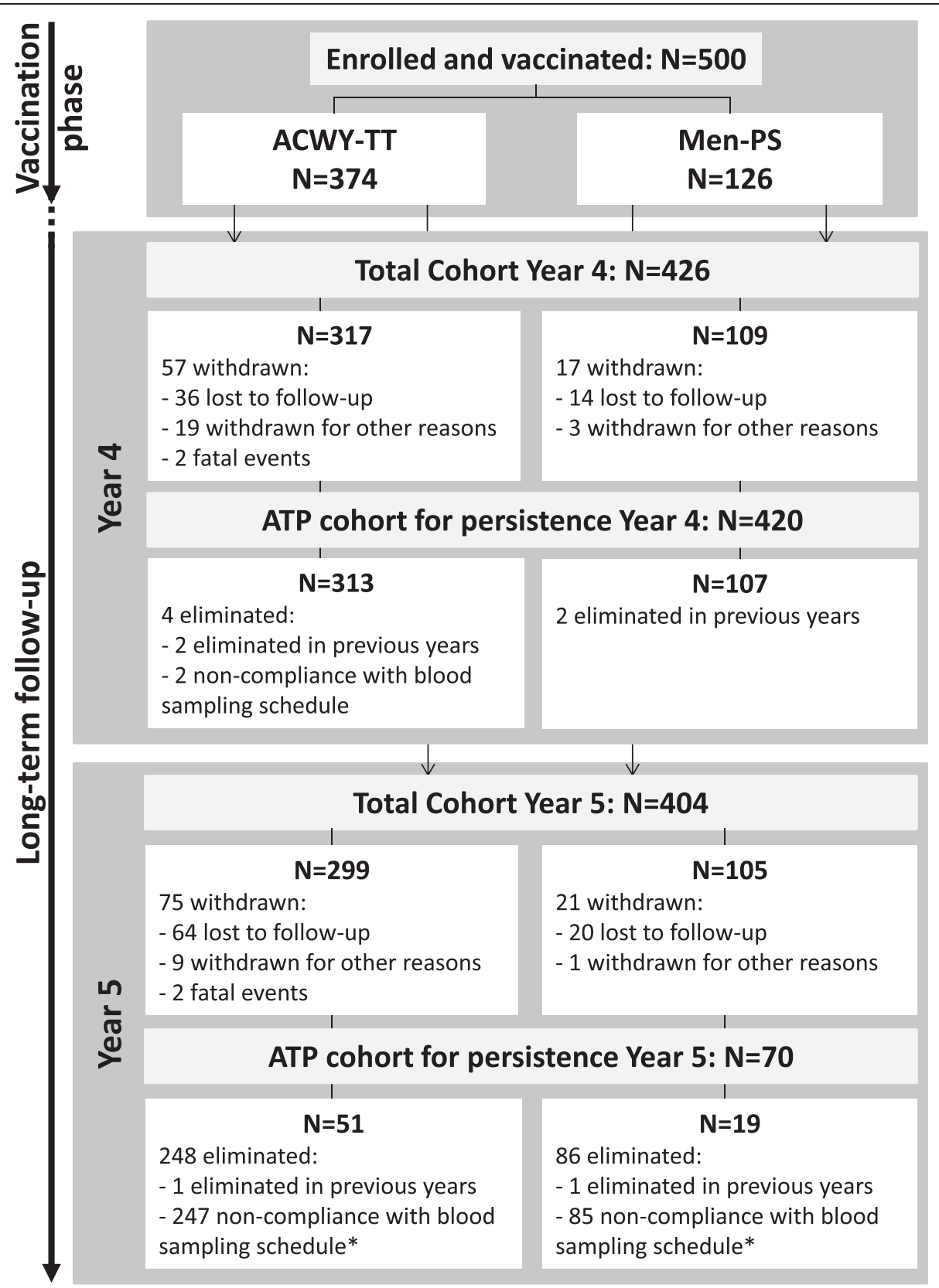

Fig. 1 Flow of participants. ACWY-TT= group of participants who received one dose of MenACWY-TT at Month 0; Men-PS=group of participants who received one dose of MenACWY-PS at Month 0; $N=$ number of participants; ATP = according to protocol. *Due to a delay in the annual approval from the FDA of the Philippines for the Year 5 persistence timepoint, all participants enrolled in the Philippines attended the Year 5 visit outside protocol-defined windows between the primary vaccination and the Year 5 blood sampling and hence were eliminated from the ATP analysis at Year 5 because of non-compliance with the blood sampling schedule

\section{Discussion}

This extension study reports the persistence of the immune response induced by a single dose of MenACWY-TT or a licensed MenACWY-PS in healthy adolescents and adults 5 years after vaccination. The majority of participants vaccinated with MenACWY-TT retained rSBA titers $\geq 1: 8$ against all four serogroups (71.6 \% [serogroup W] to $90.0 \%$ [serogroup A]) at Year 5 after vaccination, indicating that the protection continues for up to 5 years. This was observed for both the adolescent (11-17 years of age) and the adult (18-55 years of age) strata. The percentages of participants retaining seroprotective levels were observed to be lower in the MenACWY-PS recipients (ranging from $24.8 \%$ [serogroup W] to $74.3 \%$ [serogroup A]), with evidence for potential differences in seroprotective levels for serotypes $\mathrm{A}, \mathrm{C}, \mathrm{W}$, and $\mathrm{Y}$ shown in an exploratory analysis. 
Table 1 Demographic characteristics of the study participants (Total Cohort Year 5)

\begin{tabular}{llll}
\hline Characteristics & Parameter or category & ACWY-TT & $\begin{array}{c}\text { Men-PS } \\
(N=299)\end{array}$ \\
\hline Age at Year 5 (years) & & $23.3(7.7)$ & $23.6(8.1)$ \\
Age stratum (years) & mean age (SD) & $208(69.6)$ & $76(72.4)$ \\
& $11-17, n(\%)$ & $91(30.4)$ & $29(27.6)$ \\
Gender & $18-55, n(\%)$ & $136(45.5)$ & $52(49.5)$ \\
Race & Female, $n(\%)$ & $247(82.6)$ & $86(81.9)$ \\
& Asian - South East Asian heritage, $n(\%)$ & $52(17.4)$ & $19(18.1)$ \\
\hline
\end{tabular}

ACWY-TT = group of participants who received one dose of MenACWY-TT at Month 0

Men-PS = group of participants who received one dose of MenACWY-PS at Month 0

$N=$ total number of participants

$\mathrm{SD}=$ standard deviation

$n(\%)=$ number (percentage) of participants in a given category

Our persistence results are consistent with previously published data, which showed that at 3.5 years following a single dose of MenACWY-TT administered to adolescents (15-19 years old), all participants had rSBA titers $\geq 1: 8$ against all four serogroups [31]. Another study with a single dose of MenACWY-CRM in adolescents showed similar results at 5 years after primary vaccination; in the rSBA assay performed at PHE, 78 to $98 \%$ of the MenACWY-CRM recipients retained rSBA titers $\geq 1: 8$ against serogroups A, W and Y at Year 5, and only $56 \%$ of the participants against serogroup C [44].

Although IMD incidence is highest in infants, there is a secondary disease peak in adolescents [51]. In addition, the adolescent population plays an important role in the epidemiology of meningococcal disease as the main carrier group. Asymptomatic carriage is most common in adolescents and young adults [52-54], and since most transmission occurs through asymptomatic carriers, the adolescent population is an important reservoir for transmission. It was previously demonstrated that meningococcal serogroup $\mathrm{C}$ conjugate vaccines reduce asymptomatic carriage [17], therefore a vaccine that protects a high percentage of adolescents may lead to lower incidence of IMD in other age-groups.

Exploratory analyses suggested that the percentage of participants with rSBA titers $\geq 1: 8$ or $\geq 1: 128$, and the rSBA GMTs were significantly higher in the ACWY-TT group than in the Men-PS group for serogroups A, W and $Y$ at 5 years after vaccination. For serogroup $C$, the results were comparable in terms of percentages of participants with seroprotective levels and rSBA GMTs in both groups, and there was no evidence of a significant difference. This is in line with the results reported in the primary study for the Year 3 timepoint [30] and with a previous study which also showed that the rSBA GMTs of MenACWY-TT were significantly higher than those of MenACWY-PS for serogroups A, W, and Y in participants aged 18-55 years [34]. Several previous studies with all three quadrivalent conjugate vaccines (MenACWY-TT,
MenACWY-DT and MenACWY-CRM) reported evidence for higher antibody titers in the recipients of conjugate vaccines as compared to those induced by polysaccharide vaccines $[22,27,44,55,56]$. While several of these studies evaluated GMTs after a short period following vaccination, there is evidence that higher GMTs established initially correspond to higher proportions of participants retaining the protective bactericidal antibody threshold during long-term follow-up [42]. This observation is valid for the current study as well, especially for serogroup W, where a low initial GMT value in the MenPS group correlated with a low percentage of participants $(\sim 25 \%)$ who retained seroprotective rSBA-MenW titers at Year 5 postvaccination. The waning of antibody titers between 1 and 2 years post-vaccination with MenACWY-PS suggests that an earlier ( 2 years) than currently recommended ( 5 years) revaccination may be warranted in individuals who remain at high risk of exposure to $N$. meningitidis, preferably with a meningococcal conjugate vaccine.

During this study, no SAEs related to vaccination were reported and all pregnancies had normal outcomes, suggesting that the long-term safety and reactogenicity profile of MenACWY-TT was clinically acceptable. No AEs due to lack of vaccine efficacy were reported, but this needs to be interpreted in the light of the fact that no meningococcal invasive disease outbreaks have occurred in the Philippines or in Saudi Arabia since 2006 [38].

The present study was limited by its open design, which could have introduced bias in the safety reporting; however, since no safety concerns were raised in either group, this was probably not a major drawback. The study was also potentially limited by the exploratory nature of the statistical comparisons, which should be interpreted with caution, considering that the study was not designed for inferential comparisons at Year 5, there was no adjustment for multiplicity of comparisons, and the clinical relevance of these differences is unknown. Antibody titers were measured using an rSBA assay, due to the practical advantages of a commercially available 
Table 2 Percentage of participants with rSBA titers above the cut-off and GMTs (ACWY-TT and Men-PS groups)

\begin{tabular}{|c|c|c|c|c|c|c|}
\hline \multirow[t]{2}{*}{ Antibody } & \multirow[t]{2}{*}{ Estimate } & \multirow[t]{2}{*}{ Timing } & \multicolumn{2}{|c|}{ ACWY-TT } & \multicolumn{2}{|c|}{ Men-PS } \\
\hline & & & N & Value (95 \% Cl) & $N$ & Value $(95 \%$ Cl) \\
\hline \multirow[t]{18}{*}{ MenA } & \multirow[t]{6}{*}{$\% \geq 1: 8$} & Pre & 30 & $6.7(0.8,22.1)$ & 19 & $0(0.0,17.6)$ \\
\hline & & Month 1 & 30 & $100(88.4,100)$ & 19 & $100(82.4,100)$ \\
\hline & & Year 1 & 30 & $93.3(77.9,99.2)$ & 19 & $84.2(60.4,96.6)$ \\
\hline & & Year 2 & 99 & 94.9 (88.6; 98.3) & 98 & $91.8(84.5 ; 96.4)$ \\
\hline & & Year 4 & 312 & $86.5(82.2 ; 90.1)$ & 107 & $73.8(64.4 ; 81.9)$ \\
\hline & & Year 5 & 299 & $90.0(86.0,93.1)$ & 105 & $74.3(64.8,82.3)$ \\
\hline & \multirow[t]{6}{*}{$\% \geq 1: 128$} & Pre & 30 & $6.7(0.8,22.1)$ & 19 & $0(0.0,17.6)$ \\
\hline & & Month 1 & 30 & $100(88.4,100)$ & 19 & $100(82.4,100)$ \\
\hline & & Year 1 & 30 & $90.0(73.5,97.9)$ & 19 & $78.9(54.4,93.9)$ \\
\hline & & Year 2 & 99 & $92.9(86.0 ; 97.1)$ & 98 & $87.8(79.6 ; 93.5)$ \\
\hline & & Year 4 & 312 & $78.5(73.5 ; 83.0)$ & 107 & $61.7(51.8 ; 70.9)$ \\
\hline & & Year 5 & 299 & $86.3(81.9,90.0)$ & 105 & $68.6(58.8,77.3)$ \\
\hline & \multirow[t]{6}{*}{ GMT } & Pre & 30 & $2.7(1.7,4.3)$ & 19 & $2.0(2.0,2.0)$ \\
\hline & & Month 1 & 30 & $4231.2(2730.7,6556.2)$ & 19 & $1463.2(886.5,2415.0)$ \\
\hline & & Year 1 & 30 & $1066.9(472.4,2409.6)$ & 19 & $218.0(71.0,669.6)$ \\
\hline & & Year 2 & 99 & $807.1(559.5 ; 1164.2)$ & 98 & $385.8(259.4 ; 573.9)$ \\
\hline & & Year 4 & 312 & $278.6(219.7 ; 353.2)$ & 107 & $105.4(67.6 ; 164.4)$ \\
\hline & & Year 5 & 299 & $303.9(248.2,372.0)$ & 105 & $103.6(67.8,158.3)$ \\
\hline \multirow[t]{18}{*}{ MenC } & \multirow[t]{6}{*}{$\% \geq 1: 8$} & Pre & 30 & $10.0(2.1,26.5)$ & 18 & $0.0(0.0,18.5)$ \\
\hline & & Month 1 & 30 & $100(88.4,100)$ & 18 & $100(81.5,100)$ \\
\hline & & Year 1 & 30 & $96.7(82.8,99.9)$ & 17 & $94.1(71.3,99.9)$ \\
\hline & & Year 2 & 100 & $98.0(93.0 ; 99.8)$ & 99 & $86.9(78.6 ; 92.8)$ \\
\hline & & Year 4 & 312 & $88.5(84.4 ; 91.8)$ & 107 & $84.1(75.8 ; 90.5)$ \\
\hline & & Year 5 & 299 & $79.3(74.2,83.7)$ & 104 & $71.2(61.4,79.6)$ \\
\hline & \multirow[t]{6}{*}{$\% \geq 1: 128$} & Pre & 30 & $3.3(0.1,17.2)$ & 18 & $0.0(0.0,18.5)$ \\
\hline & & Month 1 & 30 & $100(88.4,100)$ & 18 & $100(81.5,100)$ \\
\hline & & Year 1 & 30 & $83.3(65.3,94.4)$ & 17 & $94.1(71.3,99.9)$ \\
\hline & & Year 2 & 100 & $86.0(77.6 ; 92.1)$ & 99 & $79.8(70.5 ; 87.2)$ \\
\hline & & Year 4 & 312 & $81.4(76.6 ; 85.6)$ & 107 & $73.8(64.4 ; 81.9)$ \\
\hline & & Year 5 & 299 & $69.2(63.7,74.4)$ & 104 & $65.4(55.4,74.4)$ \\
\hline & \multirow[t]{6}{*}{ GMT } & Pre & 30 & $3.1(1.7,5.6)$ & 18 & $2.0(2.0,2.0)$ \\
\hline & & Month 1 & 30 & $6886.0(4473.9,10598.7)$ & 18 & $8070.7(4896.6,13302.2)$ \\
\hline & & Year 1 & 30 & $462.7(239.2,895.2)$ & 17 & $1956.8(731.8,5232.7)$ \\
\hline & & Year 2 & 100 & 304.4 (232.0; 399.5) & 99 & $286.3(181.8 ; 450.9)$ \\
\hline & & Year 4 & 312 & $273.6(220.6 ; 339.4)$ & 107 & $315.0(196.8 ; 504.1)$ \\
\hline & & Year 5 & 299 & $114.0(90.5,143.5)$ & 104 & $142.4(85.3,237.6)$ \\
\hline \multirow[t]{7}{*}{ MenW } & \multirow[t]{6}{*}{$\% \geq 1: 8$} & Pre & 30 & $3.3(0.1,17.2)$ & 18 & $11.1(1.4,34.7)$ \\
\hline & & Month 1 & 30 & $96.7(82.8,99.9)$ & 17 & $76.5(50.1,93.2)$ \\
\hline & & Year 1 & 30 & $93.3(77.9,99.2)$ & 18 & $66.7(41.0,86.7)$ \\
\hline & & Year 2 & 100 & $84.0(75.3 ; 90.6)$ & 100 & $24.0(16.0 ; 33.6)$ \\
\hline & & Year 4 & 312 & $74.0(68.8 ; 78.8)$ & 107 & $25.2(17.3 ; 34.6)$ \\
\hline & & Year 5 & 299 & $71.6(66.1,76.6)$ & 105 & $24.8(16.9,34.1)$ \\
\hline & $\% \geq 1: 128$ & Pre & 30 & $0.0(0.0,11.6)$ & 18 & $5.6(0.1,27.3)$ \\
\hline
\end{tabular}


Table 2 Percentage of participants with rSBA titers above the cut-off and GMTs (ACWY-TT and Men-PS groups) (Continued)

\begin{tabular}{|c|c|c|c|c|c|c|}
\hline & & Month 1 & 30 & $96.7(82.8,99.9)$ & 17 & $76.5(50.1,93.2)$ \\
\hline & & Year 1 & 30 & $90.0(73.5,97.9)$ & 18 & $61.1(35.7,82.7)$ \\
\hline & & Year 2 & 100 & $80.0(70.8 ; 87.3)$ & 100 & $19.0(11.8 ; 28.1)$ \\
\hline & & Year 4 & 312 & $68.6(63.1 ; 73.7)$ & 107 & $20.6(13.4 ; 29.5)$ \\
\hline & & Year 5 & 299 & $64.9(59.2,70.3)$ & 105 & $21.0(13.6,30.0)$ \\
\hline & GMT & Pre & 30 & $2.1(1.9,2.5)$ & 18 & $3.3(1.6,7.0)$ \\
\hline & & Month 1 & 30 & $9571.6(4649.0,19706.4)$ & 17 & $881.6(150.8,5154.0)$ \\
\hline & & Year 1 & 30 & $1659.2(728.5,3778.7)$ & 18 & $120.3(23.6,614.5)$ \\
\hline & & Year 2 & 100 & $257.8(161.8 ; 410.7)$ & 100 & $6.5(4.2 ; 10.0)$ \\
\hline & & Year 4 & 312 & $175.1(131.5 ; 233)$ & 107 & $11.3(7.8 ; 16.3)$ \\
\hline & & Year 5 & 299 & $170.2(124.7,232.4)$ & 105 & $11.7(7.9,17.1)$ \\
\hline MenY & $\% \geq 1: 8$ & Pre & 28 & $28.6(13.2,48.7)$ & 11 & $9.1(0.2,41.3)$ \\
\hline & & Month 1 & 27 & $100(87.2,100)$ & 12 & $100(73.5,100)$ \\
\hline & & Year 1 & 28 & $96.4(81.7,99.9)$ & 12 & $50.0(21.1,78.9)$ \\
\hline & & Year 2 & 100 & $86.0(77.6 ; 92.1)$ & 100 & $44.0(34.1 ; 54.3)$ \\
\hline & & Year 4 & 309 & $82.8(78.2 ; 86.9)$ & 107 & $43.9(34.3 ; 53.9)$ \\
\hline & & Year 5 & 299 & $84.3(79.7,88.2)$ & 105 & $44.8(35.0,54.8)$ \\
\hline & $\% \geq 1: 128$ & Pre & 28 & $17.9(6.1,36.9)$ & 11 & $9.1(0.2,41.3)$ \\
\hline & & Month 1 & 27 & $100(87.2,100)$ & 12 & $100(73.5,100)$ \\
\hline & & Year 1 & 28 & $89.3(71.8,97.7)$ & 12 & $33.3(9.9,65.1)$ \\
\hline & & Year 2 & 100 & $82.0(73.1 ; 89.0)$ & 100 & $36.0(26.6 ; 46.2)$ \\
\hline & & Year 4 & 309 & $78.6(73.6 ; 83.1)$ & 107 & $34.6(25.6 ; 44.4)$ \\
\hline & & Year 5 & 299 & $80.9(76.0,85.2)$ & 105 & $41.9(32.3,51.9)$ \\
\hline & GMT & Pre & 28 & $9.3(3.5,24.5)$ & 11 & $3.4(1.2,9.7)$ \\
\hline & & Month 1 & 27 & $3659.5(2193.4,6105.6)$ & 12 & $2663.0(1821.9,3892.4)$ \\
\hline & & Year 1 & 28 & $1157.7(572.2,2342.3)$ & 12 & $22.3(3.4,146.2)$ \\
\hline & & Year 2 & 100 & $367.1(232.2 ; 580.2)$ & 100 & $19.4(11.4 ; 33.0)$ \\
\hline & & Year 4 & 309 & $350.5(268.9 ; 456.7)$ & 107 & $26.0(16.6 ; 40.7)$ \\
\hline & & Year 5 & 299 & $306.0(236.3,396.3)$ & 105 & $29.6(18.7,46.7)$ \\
\hline
\end{tabular}

Percentage of participants with rSBA antibody titers $\geq 1: 8$ and $\geq 1: 128$ and geometric mean titers in the ACWY-TT and the Men-PS groups at pre-vaccination, Month 1 and Year 1 (subset of participants from the ATP cohort for persistence Year 2 with samples tested by rSBA at the PHE laboratory) and at Years 2 (ATP cohort for persistence Year 2), 4 (ATP cohort for persistence Year 4) and 5 (Total Cohort Year 5)

ACWY-TT = group of participants who received one dose of MenACWY-TT at Month 0

Men-PS = group of participants who received one dose of MenACWY-PS at Month 0

ATP $=$ according to protocol

Pre $=$ pre-vaccination; Month $1=1$ month post-vaccination, Year 1, 2, 4 and 5=1,2, 4 and 5 years post-vaccination

$N=$ number of participants with available results

$\%=$ percentage of participants with titers within the specified range

GMT = geometric mean titer

$95 \% \mathrm{Cl}=95 \%$ confidence interval

Bold: $95 \% \mathrm{Cl}$ on group difference or GMT ratio excluding equality between the ACWY-TT and Men-PS groups

heterologous complement source. However, it is known that the use of rabbit complement as opposed to human complement results in a higher absolute value of the titers $[42,44,48]$. Finally, only a limited number of samples from pre-vaccination, Month 1, Years 1 and 2 were retested at PHE. Although a direct comparison should be made cautiously because of inter-laboratory procedural differences, different sample sizes, and a different number of freeze-thaw cycles for a given clinical sample, the re-analysis at PHE gave lower point estimates compared to the original rSBA assay performed at GSK Vaccines [30].

\section{Conclusions}

This study showed that MenACWY-TT, when administered as a single intramuscular dose to adolescents and adults 11-55 years of age, induced an immune response against meningococcal serogroups $\mathrm{A}, \mathrm{C}, \mathrm{W}$ and $\mathrm{Y}$, which 
persisted in the majority of participants up to 5 years after vaccination regardless of age stratum. The longterm immunogenicity for serogroups $\mathrm{A}, \mathrm{W}$ and $\mathrm{Y}$ was observed to be higher in the conjugated vaccine group compared to the polysaccharide vaccine group.

Menactra is a trademark of Sanofi Pasteur.

Nimenrix, Menveo, and Mencevax are trademarks of the GSK Group of companies.

\section{Additional file}

Additional file 1: Table S1. Percentage of participants with rSBA antibody titers above the cut-off and GMTs (ATP cohort for persistence Year 5). Table S2. Percentage of participants per age strata with rSBA titers above the cut-off and GMTs (Total Cohort Year 5). Percentage of participants per age strata with rSBA antibody titers $\geq 1: 8$ and $\geq 1: 128$ and geometric mean titers in the ACWY-TT and the Men-PS groups at pre-vaccination, at Years 4 and 5 (Total Cohort Year 5). (DOC $111 \mathrm{~kb}$ )

\section{Abbreviations}

ATP: According-to-protocol; Cl: Confidence interval; $\mathrm{CRM}_{197}$ : Corynebacterium diphtheriae toxin; DT: Diphtheria toxoid; FDA: Food and Drug Administration; GMT: Geometric mean antibody titer; MenACWY: Quadrivalent meningococcal vaccine; MenPS: Meningococcal polysaccharide; PHE: Public Health England; rSBA: Rabbit complement; SAE: Serious adverse event; TT: Tetanus toxoid.

\section{Competing interests}

Dr Charissa Borja-Tabora and Dr Cecilia Montalban received grants from the GSK group of companies. Devayani Kolhe and Drs Marie Van der Wielen, Dominique Boutriau and Jacqueline Miller are employees of GSK group of companies. Drs Marie Van der Wielen and Jacqueline Miller declare stock ownership in the GSK group of companies. Drs Dominique Boutriau and Jacqueline Miller are also inventors of certain patents of the GSK group of companies. Dr Ziad A. Memish declares that he has no competing interests.

\section{Authors' contributions}

$C B T, C M, Z M$, and $B C$ were involved in collection of data and interpretation of the results. $D B, J M M$ and $M W W$ were involved in interpretation of the results and coordination of the study. DB, JMM, MVW, and DK were involved in design and planning of the study. DK provided statistical expertise. All authors had full access to the data and had final responsibility to submit for publication. Drafts were developed by a professional publication writer according to the recommendations, documentation, and outline provided by the lead author. All authors read and approved the final manuscript.

\section{Authors' information}

Not applicable

\section{Availability of data and materials}

Not applicable

\section{Acknowledgments}

The Authors are indebted to the study participants and their parents, clinicians, nurses, and laboratory technicians at the study site, as well as to clinical investigators for their contribution to this study. We are also grateful to GSK Vaccines study team members for their contribution to this study and to the Vaccine Evaluation Unit, Public Health England, Manchester, UK for performing the serum bactericidal antibody assays. The Authors also thank Vasile Coman (XPE Pharma \& Science) for scientific writing support and Melissa McNeely (XPE Pharma \& Science, c/o GSK Vaccines) for publication coordination.

\section{Funding}

GlaxoSmithKline Biologicals SA was the funding source and was involved in all stages of the study conduct and analysis. GlaxoSmithKline Biologicals SA also funded all costs associated with the development and the publishing of the present manuscript.

\section{Author details}

${ }^{1}$ Research Institute for Tropical Medicine, Corporate Ave, Muntinlupa City 1781 Metro Manila, Philippines. ${ }^{2}$ Philippine General Hospital Manila, Taft Avenue, Ermita, Manila 1000Metro Manila, Philippines. ${ }^{3}$ College of Medicine, Alfaisal University, Al Zahrawi Street, Al Maather, Al Takhassusi Rd, Riyadh 11533, Saudi Arabia. ${ }^{4}$ GSK Vaccines, Avenue Fleming 20, B- 1300 Wavre, Belgium. ${ }^{5}$ GSK Pharmaceuticals Limited, Embassy Links, \#5, SRT Road, Bangalore 560052, India. 'GSK Vaccines, 2301 Renaissance Blvd, King of Prussia, PA 19406, USA.

Received: 12 February 2015 Accepted: 21 September 2015

Published online: 06 October 2015

\section{References}

1. Halperin SA, Bettinger JA, Greenwood B, Harrison LH, Jelfs J, Ladhani SN, et al. The changing and dynamic epidemiology of meningococcal disease. Vaccine. 2012;30 Suppl 2:B26-36.

2. Jafri RZ, Ali A, Messonnier NE, Tevi-Benissan C, Durrheim D, Eskola J, et al. Global epidemiology of invasive meningococcal disease. Popul Health Metr. 2013;11(1):17.

3. Harrison LH, Trotter CL, Ramsay ME. Global epidemiology of meningococcal disease. Vaccine. 2009;27 Suppl 2:B51-63.

4. Memish ZA, Goubeaud A, Broker M, Malerczyk C, Shibl AM. Invasive meningococcal disease and travel. J Infect Public Health. 2010;3(4):143-51.

5. Goodman AL, Masuet-Aumatell C, Halbert J, Zuckerman JN. Awareness of meningococcal disease among travelers from the United Kingdom to the meningitis belt in Africa. Am J Trop Med Hyg. 2014;91(2):281-6.

6. Dijkstra JA, Chappuis F, Loutan L. Vaccine-preventable diseases in long-term expatriates. J Travel Med. 2005;12 Suppl 1:S47-57.

7. Cramer JP, Wilder-Smith A. Meningococcal disease in travelers: update on vaccine options. Curr Opin Infect Dis. 2012;25(5):507-17.

8. World Health Organization: Global Alert and Response (GAR). Meningococcal disease in the Philippines - update 2. 28 January 2005 Available at: http://www.who.int/csr/don/2005 01 28a/en/.

9. Lingappa JR, Al-Rabeah AM, Hajjeh R, Mustafa T, Fatani A, Al-Bassam T, et al. Serogroup W-135 meningococcal disease during the Hajj, 2000. Emerg Infect Dis. 2003;9(6):665-71.

10. Memish ZA, Ahmed QA. Mecca bound: the challenges ahead. J Travel Med. 2002:9(4):202-10.

11. Wilder-Smith A, Goh KT, Barkham T, Paton NI. Hajj-associated outbreak strain of Neisseria meningitidis serogroup W135: estimates of the attack rate in a defined population and the risk of invasive disease developing in carriers. Clin Infect Dis. 2003;36(6):679-83.

12. Borrow R. Meningococcal disease and prevention at the Hajj. Travel Med Infect Dis. 2009;7(4):219-25.

13. Menomune: Package Insert. Available from: http://www.fda.gov/downloads/ BiologicsBloodVaccines/Vaccines/ApprovedProducts/UCM308370.pdf. [Accessed 22 January 2015].

14. Tan LK, Carlone GM, Borrow R. Advances in the development of vaccines against Neisseria meningitidis. N Engl J Med. 2010;362(16):1511-20.

15. Zahlanie YC, Hammadi MM, Ghanem ST, Dbaibo GS. Review of meningococcal vaccines with updates on immunization in adults. Hum Vaccin Immunother. 2014;10(4):995-1007.

16. Borrow $\mathrm{R}$, Miller $\mathrm{E}$. Long-term protection in children with meningococcal $\mathrm{C}$ conjugate vaccination: lessons learned. Expert Rev Vaccines. 2006;5(6):851-7.

17. Maiden MC, Ibarz-Pavon AB, Urwin R, Gray SJ, Andrews NJ, Clarke SC, et al. Impact of meningococcal serogroup $C$ conjugate vaccines on carriage and herd immunity. J Infect Dis. 2008;197(5):737-43.

18. Ramsay ME, Andrews NJ, Trotter CL, Kaczmarski EB, Miller E. Herd immunity from meningococcal serogroup C conjugate vaccination in England: database analysis. BMJ. 2003;326(7385):365-6.

19. Trotter $C L$, Maiden MC. Meningococcal vaccines and herd immunity: lessons learned from serogroup $C$ conjugate vaccination programs. Expert Rev Vaccines. 2009;8(7):851-61.

20. Richmond P, Kaczmarski E, Borrow R, Findlow J, Clark S, McCann R, et al. Meningococcal $C$ polysaccharide vaccine induces immunologic hyporesponsiveness in adults that is overcome by meningococcal $C$ conjugate vaccine. J Infect Dis. 2000;181(2):761-4. 
21. Baxter R, Baine Y, Ensor K, Bianco V, Friedland LR, Miller JM. Immunogenicity and safety of an investigational quadrivalent meningococcal ACWY tetanus toxoid conjugate vaccine in healthy adolescents and young adults 10 to 25 years of age. Pediatr Infect Dis J. 2011;30(3):e41-8.

22. Bermal N, Huang LM, Dubey AP, Jain H, Bavdekar A, Lin TY, et al. Safety and immunogenicity of a tetravalent meningococcal serogroups $A, C, W-135$ and $Y$ conjugate vaccine in adolescents and adults. Hum Vaccin. 2011;7(2):239-47.

23. Knuf M, Baine $Y$, Bianco V, Boutriau D, Miller JM. Antibody persistence and immune memory 15 months after priming with an investigational tetravalent meningococcal tetanus toxoid conjugate vaccine (MenACWY-TT) in toddlers and young children. Hum Vaccin Immunother. 2012;8(7):866-72.

24. Knuf $M$, Kieninger-Baum D, Habermehl $P$, Muttonen $P$, Maurer $H$, Vink $P$, et al. A dose-range study assessing immunogenicity and safety of one dose of a new candidate meningococcal serogroups A, C, W-135, Y tetanus toxoid conjugate (MenACWY-TT) vaccine administered in the second year of life and in young children. Vaccine. 2010;28(3):744-53.

25. Knuf M, Pantazi-Chatzikonstantinou A, Pfletschinger U, Tichmann-Schumann I, Maurer $\mathrm{H}$, Maurer L, et al. An investigational tetravalent meningococcal serogroups A, C, W-135 and Y-tetanus toxoid conjugate vaccine COadministered with Infanrix hexa is immunogenic, with an acceptable safety profile in 12-23-month-old children. Vaccine. 2011;29(25):4264-73.

26. Memish ZA, Dbaibo G, Montellano M, Verghese VP, Jain H, Dubey AP, et al. Immunogenicity of a single dose of tetravalent meningococcal serogroups A, C, W-135, and Y conjugate vaccine administered to 2- to 10-year-olds is noninferior to a licensed-ACWY polysaccharide vaccine with an acceptable safety profile. Pediatr Infect Dis J. 2011;30(4):e56-62.

27. Ostergaard L, Lebacq E, Poolman J, Maechler G, Boutriau D. Immunogenicity, reactogenicity and persistence of meningococcal A, C, W-135 and Y-tetanus toxoid candidate conjugate (MenACWY-TT) vaccine formulations in adolescents aged 15-25 years. Vaccine. 2009;27(1):161-8.

28. Vesikari T, Karvonen A, Bianco V, Van der Wielen M, Miller J. Tetravalent meningococcal serogroups $A, C, W-135$ and $Y$ conjugate vaccine is well tolerated and immunogenic when co-administered with measles-mumpsrubella-varicella vaccine during the second year of life: An open, randomized controlled trial. Vaccine. 2011;29(25):4274-84.

29. Findlow $\mathrm{H}$, Borrow R. Immunogenicity and safety of a meningococcal serogroup A, C, Y and W glycoconjugate vaccine, ACWY-TT. Adv Ther. 2013:30(5):431-58.

30. Borja-Tabora C, Montalban C, Memish ZA, Van der Wielen M, Bianco V Boutriau D, et al. Immune response, antibody persistence, and safety of a single dose of the quadrivalent meningococcal serogroups $A, C$, $W-135$, and $Y$ tetanus toxoid conjugate vaccine in adolescents and adults: results of an open, randomised, controlled study. BMC Infect Dis. 2013;13:116.

31. Ostergaard L, Van der Wielen M, Bianco V, Miller JM. Persistence of antibodies for 42 months following vaccination of adolescents with a meningococcal serogroups A, C, W-135, and $Y$ tetanus toxoid conjugate vaccine (MenACWY-TT). Int J Infect Dis. 2013;17(3):e173-6.

32. Vesikari T, Forsten A, Boutriau D, Bianco V, Van der Wielen M, Miller JM. A randomized study to assess the immunogenicity, antibody persistence and safety of a tetravalent meningococcal serogroups A, C, W-135 and Y tetanus toxoid conjugate vaccine in children aged $2-10$ years. Hum Vaccin Immunother. 2012;8(12):1882-91.

33. Vesikari T, Forsten A, Boutriau D, Bianco V, Van der Wielen M, Miller JM. Randomized trial to assess the immunogenicity, safety and antibody persistence up to three years after a single dose of a tetravalent meningococcal serogroups A, C, W-135 and Y tetanus toxoid conjugate vaccine in toddlers. Hum Vaccin Immunother. 2012;8(12):1892-903.

34. Dbaibo G, Macalalad N, Aplasca-De Los Reyes MR, Dimaano E, Bianco V, Baine $Y$, et al. The immunogenicity and safety of an investigational meningococcal serogroups $A, C, W-135, Y$ tetanus toxoid conjugate vaccine (ACWY-TT) compared with a licensed meningococcal tetravalent polysaccharide vaccine: a randomized, controlled non-inferiority study. Hum Vaccin Immunother. 2012;8(7):873-80.

35. Vesikari T, Forsten A, Bianco V, Van Der Wielen M, Miller JM. Antibody persistence to meningococcal serogroups $A, C, W$ and $y$ in toddlers two years after vaccination with a quadrivalent meningococcal ACWY-tetanus toxoid conjugate (MenACWY-TT) vaccine as measured by bactericidal antibody assays using rabbit or human complement. Trials in Vaccinology. 2014:3(1):121-6.
36. Memish Z, Al Hakeem R, Al Neel O, Danis K, Jasir A, Eibach D. Laboratoryconfirmed invasive meningococcal disease: effect of the Hajj vaccination policy, Saudi Arabia, 1995 to 2011. Euro Surveill. 2013;18:37.

37. Memish ZA. Health conditions for travelers to Saudi Arabia for (Hajj) for the year 1431H/2010. J Infect Public Health. 2010;3(3):92-4.

38. World Health Organization: Global Alert and Response (GAR). Meningococcal disease. [http://www.who.int/csr/don/archive/disease/ meningococcal_disease/en/].

39. World Health Organization: WHO vaccine-preventable diseases: monitoring system. 2013 global summary. [http://apps.who.int/ immunization_monitoring/globalsummary].

40. Steffen $\mathrm{R}$. The risk of meningococcal disease in travelers and current recommendations for prevention. J Travel Med. 2010;17(Suppl):9-17.

41. Philippine Foundation for Vaccination (PFV) [http://www.philvaccine.org/ vaccination-schedules/].

42. Gill CJ, Baxter R, Anemona A, Ciavarro G, Dull P. Persistence of immune responses after a single dose of Novartis meningococcal serogroup $A, C$, W-135 and Y CRM-197 conjugate vaccine (Menveo(R)) or Menactra(R) among healthy adolescents. Hum Vaccin. 2010;6(11):881-7.

43. Vu DM, Welsch JA, Zuno-Mitchell P, Dela Cruz JV, Granoff DM. Antibody persistence 3 years after immunization of adolescents with quadrivalent meningococcal conjugate vaccine. J Infect Dis. 2006;193(6):821-8.

44. Jacobson RM, Jackson LA, Reisinger K, Izu A, Odrljin T, Dull PM. Antibody persistence and response to a booster dose of a quadrivalent conjugate vaccine for meningococcal disease in adolescents. Pediatr Infect Dis J. 2013;32(4):e170-7.

45. Auckland C, Gray S, Borrow R, Andrews N, Goldblatt D, Ramsay M, et al. Clinical and immunologic risk factors for meningococcal $\mathrm{C}$ conjugate vaccine failure in the United Kingdom. J Infect Dis. 2006;194(12):1745-52.

46. Maslanka SE, Gheesling LL, Libutti DE, Donaldson KB, Harakeh HS, Dykes JK, et al. Standardization and a multilaboratory comparison of Neisseria meningitidis serogroup A and C serum bactericidal assays. The Multilaboratory Study Group. Clin Diagn Lab Immunol. 1997:4(2):156-67.

47. Borrow R, Balmer P, Miller E. Meningococcal surrogates of protection-serum bactericidal antibody activity. Vaccine. 2005;23(17-18):2222-7.

48. Borrow R, Andrews N, Goldblatt D, Miller E. Serological basis for use of meningococcal serogroup $C$ conjugate vaccines in the United Kingdom: reevaluation of correlates of protection. Infect Immun. 2001;69(3):1568-73.

49. Clopper CP, Pearson ES. The use of confidence or fiducial limits illustrated in the case of the binomial. Biometrika. 1934;26(4):404-13.

50. Newcombe RG. Two-sided confidence intervals for the single proportion: comparison of seven methods. Statistics in Medicine. 1998:17(8):857-72.

51. European Centre for Disease Control and Prevention. Annual epidemiological report 2014 - Vaccine-preventable diseases - invasive bacterial diseases. Stockholm: ECDC; 2015. Accesible at: http:// ecdc.europa.eu/en/publications/Publications/AER-VPD-IBD-2014.pdf

52. MacLennan J, Kafatos G, Neal K, Andrews N, Cameron JC, Roberts R, et al. Social Behavior and Meningococcal Carriage in British Teenagers. Emerg Infect Dis. 2006;12(6):950-7.

53. Christensen $H$, May M, Bowen L, Hickman M, Trotter CL. Meningococcal carriage by age: a systematic review and meta-analysis. Lancet Infect Dis. 2010;10(12):853-61.

54. Soriano-Gabarro M, Wolter J, Hogea C, Vyse A. Carriage of Neisseria meningitidis in Europe: a review of studies undertaken in the region. Expert Rev Anti Infect Ther. 2011;9(9):761-74.

55. Jackson LA, Jacobson RM, Reisinger KS, Anemona A, Danzig LE, Dull PM. A randomized trial to determine the tolerability and immunogenicity of a quadrivalent meningococcal glycoconjugate vaccine in healthy adolescents. Pediatr Infect Dis J. 2009;28(2):86-91.

56. Keyserling H, Papa T, Koranyi K, Ryall R, Bassily E, Bybel MJ, et al. Safety, immunogenicity, and immune memory of a novel meningococcal (groups $A, C, Y$, and $W$-135) polysaccharide diphtheria toxoid conjugate vaccine (MCV-4) in healthy adolescents. Arch Pediatr Adolesc Med. 2005:159(10):907-13. 